\title{
Forschungsgruppe REMEMBER (Hg.) (2020). Erinnerung an den Holocaust im Religionsunterricht. Empirische Einblicke und didaktische Impulse. Stuttgart: Kohlhammer. ISBN 978-3-I 7-0389 I 2-0. 275 Seiten.
}

\author{
Rebecca Hedenkamp \\ Carl von Ossietzky Universität Oldenburg (rebecca.hedenkamp@uni-oldenburg.de)
}

Die seit 2014 aus Wissenschaftler*innen von Universitäten und Hochschulen in Tübingen, Zürich, Wien, Mainz, Freiburg und München bestehende Forschungsgruppe REMEMBER stellt in dieser Veröffentlichung die Ergebnisse ihrer Studie vor, die die Frage verfolgte, ob „Erinnerung an den Holocaust/an die Shoah" für Religionslehrkräfte ein Thema in ihrem Unterricht sei. An der 2016 gestarteten Studie haben im Rahmen einer Online-Befragung in einem Zeitraum von 12 Monaten 1204 Lehrkräfte teilgenommen.

Betrachtet man die Ausgangslage, wird die Dringlichkeit der Studie deutlich. Schon die Hinführung fordert mit Nachdruck, die religiösen Wurzeln der Shoah zu thematisieren und innerhalb des Religionsunterrichts das Verhältnis von Judentum und Christentum kritisch zu beleuchten. Innerhalb des Kontextkapitels zeigt die Gruppe anschließend auf, an welchen religionspädagogischen Forschungsstand sie, bezüglich der Thematisierung der Shoah innerhalb des Religionsunterrichts, anknüpfen kann. Dabei wird deutlich, dass dort auf theoretischer Ebene schon seit den 1980ern ein Wandel stattgefunden hat - hin zu einer „erinnerungsgeleiteten religiösen Bildung“ (29), in der die Forderung Adornos nach einer Erziehung nach Auschwitz aufgegriffen und pädagogisch reflektiert wurde. Es wird aber auch auf die Leerstelle aufmerksam gemacht: die fehlende Verbindung zwischen den theoretischen Überlegungen und der praktischen Lernwelt, in der sich sowohl Lehrende als auch Lernende aktuell befinden. Somit sieht sich die Studie auch als „Bestandsaufnahme der Situation des Religionsunterrichts“ (30) diese Explorativität des Vorhabens wird innerhalb der Veröffentlichung mehrfach angesprochen.

Die Studie an sich besteht, wie schon erwähnt, aus einem Online-Fragebogen, der durch verschiedene Kanäle an Lehrkräfte in Deutschland, Österreich und der Schweiz geleitet wurde (im Ausblick wird angeregt, dass mit diesem Verfahren auch weitere Länder derlei Forschung anstoßen könnten). Die Auswertung wird anschließend länderweise getrennt und auf die unterschiedlichen schulischen Gegebenheiten bezogen, sodass eine Art Länderprofil entsteht. Dieses Profil wird später in kurzen Kapiteln mit Lehrplänen und Curricula der einzelnen Länder ins Gespräch gebracht. Des Weiteren wird in Aufsätzen über den Materialeinsatz innerhalb des Unterrichts, die Wahrnehmung von Widerständen und die antisemitismuskritische Bildungsperspektive des Religionsunterrichts diskutiert.

Ergeben haben sich einige interessante Punkte: So ist deutlich geworden, dass die Thematisierung der Shoah in Schulen oft nur aufgrund motivierter Lehrkräfte geschieht, da „Holocaust-Education“ als inhaltliche Kompetenz weder in länderspezifischen, noch in schulinternen Lehrplänen ausdrücklich vertreten ist. Diese Diskrepanz wird immer wieder angebracht und innerhalb der Ausführungen auch als Kritikpunkt am öffentlichen Umgang mit Holocaust-Education verwendet. Die Lehrkräfte werden innerhalb des Fragebogens ganz konkret nach dem Grund ihrer Motivation bzw. nach der Relevanz des Themas gefragt. Es folgt die Auswertung und Feststellung, dass viele der Lehrkräfte, wie schon angenommen, ihre Lehrentscheidungen stark über persönliche Identifikation mit der Thematik rechtfertigen. Damit wird die angesprochene Diskrepanz zwischen Öffentlichkeit und individuellem Interesse aufgegriffen, von den Autoren stark problematisiert und mit einer Forderung nach anderen Rechtfertigungsnarrativen, die auch von der Bildungspolitik selbst geleistet werden müssten, in den Fokus gerückt. 
Widerstände, auf die Lehrkräfte bei der Thematisierung stoßen, seien dabei vor allem aus der Schülerschaft zu spüren - aufgrund Gleichgültigkeit, Übersättigung oder Abwehr, aufgrund zeitlicher Distanz oder politischer Meinungen, die konträr zur Holocaust-Education verlaufen. Die Forschungsgruppe betont demnach die erhöhte Subjektorientierung des Faches und führt die emotionale Kompetenz an, die im Religionsunterricht gefördert werden sollte. Durch die Thematisierung der Shoah würde dies in besonderem Maße ermöglicht werden.

Antisemitismus wiederum sei ein Thema, das größerer Zuwendung bedarf, da es von Lehrkräften noch ein wenig stiefmütterlich behandelt werde. Einerseits werde von den meisten die Wichtigkeit dieses Themas betont, andererseits aber auch deutlich, dass dabei oft nur der historische Antisemitismus im Blick sei und eine Beschäftigung mit aktuellen Formen eher weniger erfolge. Dies sei jedoch auch auf bildungspolitische Zusammenhänge zurückzuführen, so die Gruppe.

Dies kann auch im Kontext dessen betrachtet werden, dass viele der Lehrkräfte die Rolle des Religionsunterrichts innerhalb der Menschenrechtsbildung betonen. Sie sehen ihr Fach dabei sogar in einer besonderen Verantwortung. Diese Perspektive vertrete der Religionsunterricht durch seine elementaren Fragen an das Menschsein dabei in ausgesprochener Weise. REMEMBER betont dies im Anschluss an die Ergebnisse und macht deutlich, welchen Stellenwert das Fach innerhalb der Holocaust-Education einnehmen kann: Beginnend bei der Aufarbeitung des christlich-jüdischen Verhältnisses, hin zur persönlichen Auseinandersetzung mit der eigenen Verantwortlichkeit innerhalb eines aktuellen Dialogs.

Ein wenig behäbig geht die Gruppe mit dem Thema der Migrationsgesellschaft um. Einerseits wird diskutiert, welche Auswirkungen heterogene Lerngruppen auf die Auseinandersetzung mit der Shoah haben, andererseits werden einige problematische Schüleraussagen unkommentiert in der Studie zitiert (75). Das mag dem Anliegen der Veröffentlichung geschuldet sein - handelt es sich ausgewiesenermaßen um eine Bestandsaufnahme.

Ebenso geht aus der Studie hervor, dass sich Religionslehrkräfte nur unzureichend auf die Herausforderungen, die zunehmend heterogeneren Lerngruppen mit ihren ebenfalls zunehmend heterogenen soziokulturellen Lebenswelten an sie stellen, vorbereitet fühlen. Es macht jedoch den Anschein, als ringe REMEMBER mit diesen aktuellen Herausforderungen und finde keine richtigen Lösungsansätze in der eigenen Zunft, außer die Lehrkräftebildung zu stärken, um Antisemitismusprävention in Schulen zu erleichtern. Sie marginalisiert dabei die Rolle der christlichen Religionspädagogik, in der es versäumt wird, den Holocaust und seine Auswirkungen auf den Unterricht reflektiert aufzuarbeiten.

Als Lösungsansatz wird innerhalb der, das Buch abschließenden, religionspädagogischen Konsequenzen die Aufgabe, Hilfestellungen im Umgang mit heterogenen Lerngruppen zu entwickeln, abgegeben und dazu angeregt, mit der islamischen Religionspädagogik in die Diskussion zu treten, um Lösungen aufzeigen zu können. Dies macht natürlich Sinn, zeigt jedoch ebenfalls auf, dass hier noch großer Handlungsbedarf vor allem in der christlichen Religionspädagogik besteht.

Abschließend soll eine Empfehlung zur Lektüre der Studie ausgesprochen werden. REMEMBER hat gezeigt, welche Dringlichkeit und Relevanz das Thema Erinnerung an den Holocaust gerade für den Religionsunterricht mit sich bringt. Sie haben einen grundlegenden Rahmen geschaffen und laden nun Forscher*innen ein, diesen Rahmen zu nutzen, um weitere Ideen zu bilden und somit dieser Frage eine größere Bühne innerhalb des religionspädagogischen Diskurses zukommen zu lassen. Sie positionieren sich klar dazu, Lehrpläne zu überarbeiten und setzen sich dafür ein, dass die besondere Rolle des Religionsunterrichts in der Bildung nach Auschwitz auch auf bildungspolitischer Ebene sichtbar wird. Lehrkräfte können diese Studie außerdem dazu nutzen, um ihre eigene Lehrtätigkeit zu reflektieren und neue Impulse mit in die Praxis zu nehmen. 